\title{
The Elementary Divisors, Associated with 0 , of a Singular M-matrix
}

\author{
By Hans Schneider
}

(Received 27th Seplember 1954.)

1. Many investigations have been concerned with a squaro matrix $P$ with non-negative cocfficients (elements). It is remarkable that many interesting properties of $P$ are determined by the set $\Sigma$ of index pairs of positive (i.e. non-zcro) coefficients of $P$, the actual values of these coefficients being irrelevant. Thus, for example, the number of characteristic roots equal in absolute value to the largest non-negative characteristic root $\rho$ depends on $\Sigma$ alone, if $P$ is irreduc. ible. If $P$ is reducible, then $\Sigma$ determines the standard forms of $P$ (cf. $\S 3$ ). The multiplicity of $\rho$ depends on $\Sigma$, and on the set $S$ of

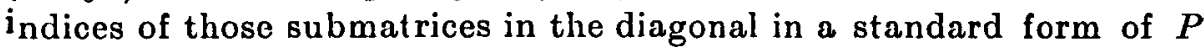
which have $\rho$ as a characteristic root. It has apparently not been considered before whether $\Sigma$ and $S$ also determine the elementary divisors associated with $\rho$. IVe shall show that, in general, the elementary divisors do not depend on these sets alone, but that necessary and sufficient conditions may be found in terms of $\Sigma$ and $S$ (a) for the elementary divisors associated with $\rho$ to be simple, and (b) that there is only one elementary divisor associated with $\rho$.

The square matrix $A=\left[a_{i j}\right]$ is called an M-matrix ${ }^{1}$ if (l) $a_{i i} \geqq 0$ for all $i$; (2) $a_{i j} \leqq 0$ when $i \neq j$; and (3) all non-zero characteristic roots of $A$ have positive real part. If $P=\left[p_{i j}\right]$ is a square matrix with non-negative coefficients and $\rho$ is its greatest non-negative characteristic root, then $\rho \geqq p_{i i}$, for all $i$ (0. Taussky [i]). Hence $\rho I-P$ is a singular M-matrix. Conversely, if $A$ is a singular M-matrix and $\rho \geqq m_{i i}$ for all $i$, then $\rho I-A$ is a matrix with nonnegative elements. Thus it is equivalent, and rather more convenient, to study the elementary divisors associated with the characteristic root 0 of a singular M-matrix.

2. We shall now explain our notation and terminology, which differ in some respects from the usual ones. We introduce a partial ordering on a set of conformable matrices with real coefficients by setting $A$ ( $\geqq B$ if $a_{i j} \geqq b_{i j}$ for all $i, j$, where $A=\left[a_{i j}\right]$ and $B=\left[b_{i j}\right]$. A second partial ordering is introduced by setting $A \geqq B$, if either

1 The term M.determinant was used by A. Ostrowski [4], [5]. It has been proved [6], p. 19, that our definition is equivalent to Ostrowaki's. 
$a_{i j}>b_{i j}$ for all $i, j$, or $A=B$. Expressions such as $A(>B$, and $A<B$ then have their natural meanings. If $A>0$, we call $A$ "strictly positive," if $A$ ( $>0$ we call $A$ " weakly positive" or just "positive." We shall similarly call $A$ negative if $A<) 0$.

The notation used by previous authors (cf. Frobenius [2], Wielandt [8], and others) is less convenient and a little less satisfactory logically. It obscures the fact that we are continually dealing with two partial orderings. While $A>B$ has the same meaning in both notations, these authors use $A \geqq B$ in place of $A$ ( $\geqq B$. Where we may write " $A$ (>0," they would havo to write " $A \geqq 0$ but $A \neq 0$." It is surely unfortunate, also, that in their notation " $A \geqq B$ " is not equivalent to " either $A>B$ or $A=B$."

We note that if $A=[a]$ is $1 \times 1$, then $A>0$ is equivalent to $A$ ( $>0$, and if $A$ is identified with $a$ then $A>0$ has its usual meaning.

Column and row vectors may be regarded as matrices, and the same notation will be employed there.

3. Our principal results will be enumerated in terms of the numbers $R_{i j}(A, P)$ defined below. Let $A$ be a square matrix ${ }^{1}$ and let $P$ be the diagonaliy symmetric partition $\left[A_{i j}\right], i, j=1, \ldots, k$. For $i, j=1, \ldots, k$ we set

and

$$
\begin{aligned}
& r_{i j}(A, P)=0 \text { if } i \neq j \text { and } A_{i j}=0, \\
& r_{i j}(A, P)=1 \text { if } i=j, \text { or if } A_{i j} \neq 0 .
\end{aligned}
$$

Where no confusion can arise we shall write $r_{i j}$ for $r_{i j}(A, P)$. Next we set

$$
R_{i j}(A, P)=\max r_{i h} r_{h l} \ldots r_{n j},
$$

the maximum being taken over all sequences $(i, h, \ldots, n, j)$. Again we shall generally write $R_{i j}$ for $R_{i j}(A, P)$. For future reference we note that

$$
\begin{gathered}
\text { either } R_{i j}=0 \text { or } R_{i j}=1 ; \\
R_{i i}=1 \text { for } i=1, \ldots, k ; \\
\sum_{h=1}^{k} R_{i h} R_{h j} \geqq R_{i j} \geqq R_{i l} R_{l j}, \quad 1 \leqq l \leqq k ; \\
\sum_{h=1, h \pm i}^{k} r_{i h} R_{h j} \geqq R_{i j} \geqq \max _{h \pm i} r_{i h} R_{h j} \quad \text { if } i \neq j .
\end{gathered}
$$

1 The field of the coefficients of $A$ is here immaterial. But in the remaining sections we shall assume that all matrices occurring have real coefficients. 
If $A$ is a square matrix, then there exists a permutation matrix, $T$, for which $A^{*}=T^{-1} A T$ can be partitioned $\left[A_{i j}^{*}\right], i, j=1, \ldots, k$ such that (1) $A_{i j}^{*}=0$ if $i<j$, (2) $A_{i i}^{*}, i=1, \ldots, k$, is irreducible. Wo shall call $A^{*}$ a standard form of $A$, and we shall say that $A^{*}$ is in standard form. In general, the standard form of $A$ is not unique. If $A^{0}$ is a standard form of $A$, and $A_{i i}^{0}, i=1, \ldots, l$, are the irreducible matrices in its diagonal, then $l=k$, and $A_{\sigma(i) \sigma(j)}^{0}=T_{i}^{-1} A_{i j}^{*} T_{j}$, where $\sigma$ is a permutation of $(1, \ldots, k)$ and $T_{1}, \ldots, T_{k}$ are permutation matrices. Thus there exists a one-one correspondence between the irreducible submatrices in the diagonal of any two standard forms such that corresponding submatrices have the same characteristic roots. In particular, all standard forms have the same number of singular irreducible submatrices in the diagonal.

In view of what is to follow we shall examine the connection between the $R_{i j}(A, P)$ and a standard form of $A$.

Lemma 1. Let $P$ be the partition $\left[A_{i j}\right], i, j=1, \ldots, k$ of the square matrix $A$ such that the $A_{i i}$ in the diagonal are irreducible. Then $A$ is in standard form if and only if $R_{i j}=0$ whenever $i<j$.

Proof. We must show that ' $R_{i j}=0$ whenever $i<j$ ' is equivalent to ' $r_{i j}=0$ whenever $i<j$ '. Clearly ' $R_{i j}=0$ whenever $i<j$ ' implies ' $r_{i j}=0$ whenever $i<j$ '. To prove the converse we note that if $i<j$, then any sequence $(i, h, \ldots, n, j)$ contains two consecutive members $l, m$ such that $l<m$. The lemma follows from the definition of $R_{i j}$.

Th EOREM 1. Let $P$ be the parti:ion $\left[A_{i j}\right], i, j=1, \ldots, k$ of $A$, where the $A_{i i}$ are irreducible, and let $A$ be in standard form. Let $1 \leqslant \alpha, \beta \leqslant k$. There exists a permulation $\sigma$ of $(1, \ldots, k)$ for which $A^{*}=\left[A_{\sigma(i) \sigma(j)}\right]$, $i, j=1, \ldots, k$, is in slandard form and $\sigma(\beta)<\sigma(\alpha)$ if and only if $R_{\beta a}(A, P)=0$.

Proof. Let $P^{*}$ be the partition $\left[A_{\sigma(i) \sigma(j .]}\right]$ of $A^{*}$ and put $R_{i j}^{*}=R_{i j}\left(A^{*}, P^{*}\right)$. We have $R_{\sigma(i) \sigma(j)}^{*}=R_{i j}=R_{i j}(A, P), i, j=1, \ldots, k$. Hence by Lemma 1 , if $A^{*}$ is in standard form and $\sigma(\beta)<\sigma(a)$, then $R_{\beta a}=R_{\left.\sigma(\beta) \sigma^{\prime} \alpha\right)}=0$.

Conversely let $R_{\beta a}=0$. Since by (1) $R_{\beta \alpha} \geqslant R_{\beta i} R_{i \mathrm{a}}$ it follows that $R_{\beta i} R_{i a}=0$ for $i=1, \ldots, k$. Hence we may partition $(1, \ldots, k)$ into three sets $E_{1}, E_{2}, E_{3}$ so that $i_{\epsilon} E_{1}$ if $l_{\beta i}=1$ and $R_{i a}=0 ; i \in E_{2}$ if $R_{\beta i}=R_{i \alpha}=0$; and $i \in E_{3}$ if $R_{\beta i}=0$ and $R_{i a}=1$. Let $\sigma$ be the permutation of $(1, \ldots, k)$ for which $\sigma(i)<\sigma(j)$ if $i<j$ and $i \in E_{\lambda}, j \in E_{\mu}$, with 
The Elementary Divisors, Associated with 0, of a Sisqular $11 \mathrm{t}$ M-Matrix

$\lambda<\mu$; while $\sigma(i)<\sigma(j)$ if $i, j \in E_{\lambda}$ and $i<j$, where $\lambda=1,2,3$. Let $P^{*}$ be the partition $\left[A_{\sigma(i) \sigma(j}\right], i, j=1, \ldots, k$, of $A^{*}$. Let $R_{i j}^{*}=R_{i j}\left(A^{*}, P^{*}\right)$. Let $i \in E_{\lambda}, j \in E_{\mu}$, and suppose that $\sigma(i)<\sigma(j)$. Then $\lambda \leqq \mu$. If $\lambda=\mu$, then $i<j$ so that by Lemma $1, R_{\sigma(i) \sigma(j)}^{*}=R_{i j}=0$. If, on the other hand, $\lambda<\mu$, then either $\lambda=1$ or $\mu=3$. If $\lambda=1$, then $R_{\beta i}=1$, whence $R_{i j}=R_{\beta i} R_{i j} \leqq R_{\beta j}=0$, since $\mu \geqq 2$. If $\mu=3$, then $R_{j a}=1$, whence $R_{i j}=R_{i i} R_{j a} \leqq R_{i a}=0$, since $\lambda \leqq 2$. Wo conclude that $R_{\sigma(i) \sigma(j)}=R_{i j}=0$ whenever $\sigma(i)<\sigma(j)$. Thus, by Lemma $1, A^{*}$ is in standard form. We need now only prove that $\sigma(\beta)<\sigma(a)$. But $R_{\beta \beta}=1, R_{\beta a}=0$, $R_{a a}=1$ imply that $\beta \epsilon E_{1}, a \in E_{3}$, and the result follows.

4. We now turn to the consideration of M-matrices. If the matrix $A$ is partitioned $\left[A_{i j}\right], i, j,=1, \ldots, k$, we shall assume any column vector $x$ to be conformably partitioned into $\left(x_{1}, \ldots, x_{k}\right)$.

Lemma 2. Let $A=\left[A_{i j}\right], i, j=1, \ldots, k$ be an $M$-matrix in standard form. Let $x=\left(x_{1}, \ldots, x_{k}\right)$ and let

$$
\left.\begin{array}{c}
x_{i}=0 \text { when } R_{i a}=0 \\
x_{i}>0 \text { when } R_{i a}=1,
\end{array}\right\}
$$

for $i=1, \ldots, h-1$, where $h>a$. If

$$
\left.\begin{array}{l}
y_{1}=0 \\
y_{i}=-\sum_{j=1}^{i-1} A_{i j} x_{j}, i=2, \ldots, k
\end{array}\right\}
$$

then

$$
\left.\begin{array}{l}
y_{h}=0 \quad \text { if } R_{h a}=0 \\
y_{h}\left(>0 \quad \text { if } R_{h a}=1\right.
\end{array}\right\} \text {. }
$$

Proof. Clearly $y_{h}\left(\geqq 0\right.$; and $y_{h}=0$ if and only if $A_{h j} x_{j}=0$ for $j=1, \ldots, h-1$, since $\left.A_{h j} x_{j} \leqq\right) 0$ for $j=1, \ldots, h-1$. Hence, by tho assumptions about the $x_{j}, y_{h}=0$ if and only if

$$
r_{h j} R_{j \mathrm{a}}=0, j=1, \ldots, h-1 \text {. }
$$

Since $r_{h j}=0$ when $h<j$ we have $\sum_{j=1}^{h-1} r_{h j} R_{j a}=\sum_{j=1, j \neq h}^{k} r_{h j} R_{j a}$ and $\max _{j<h} r_{h j} R_{j a}=\max _{j \pm h} r_{h j} R_{j a}$. Since $h \neq \alpha$, it can now easily be shown from (2) that (6) holds if and only if $R_{h a}=0$. The lemma follows.

ThEOREM 2. Let $A=\left[A_{i j}\right], i, j=1, \ldots, k$ be a singular $M$-matrix in standard form. Let $S$ be the se' of indices of singular $A_{i i}$. If $a \in S$, and 
$R_{\beta a}=0$ whenever $\beta \epsilon S, \beta \neq a$, then there exists a positive characteristic column vector $x$ of $A$ associated wi!h 0 satisfying (3) for $i=1, \ldots, k$.

Proof. Let $x$ be any column vector, and let $y$ satisfy (4). Then $A x=0$ if and only if

for $i=1, \ldots, k$.

$$
A_{i i} x_{i}=y_{i}
$$

Now let $x_{i}=0$ when $i<a$. The singular irreducible M-matrix $A_{a a}$ has a strictly positive characteristic vector $x_{a}$ associated with 0 , cf. [2], [4]. As $A$ is in standard form, $R_{i n}=0$ when $i<\alpha, R_{a a}=1$, and therefore $x_{1}, \ldots, x_{a}$ satisfy (3).

Let us suppose inductively that $x_{1}, \ldots, x_{h-1}, h>a$, satisfy (3). If $y_{1}, \ldots, y_{h}$ eatisfy (4), then $y_{h}$ also satisfies (5) by Lemma 2. Thus if $R_{h a}=0$ then $y_{h}=0$; and so if $x_{h}=0$ then $x_{h}$ satisfies (7) for $i=h$. If $R_{h a}=1$, then $y_{h}\left(>0\right.$, and by assumption $A_{h h}$ is non-singular. It is known that the inverse of a non-singular irreducible M-matrix is strictly positive ([2], [4]). Hence if $x_{h}=A_{h h}^{-1} y_{h}$, then $x_{h}>0$, and $x_{h}$ satisfies (7). We have thus constructed a vector $x_{h}$ satisfying (3) and (7), for $i=h$. The theorem follows by induction.

For the sake of completeness we shall prove the well-known Corollary 1.

Corollary 1. A singular M-matrix has a positive characteristic vector associaled with 0 .

Proof. Let $\alpha$ be the largest member of $S$. Then $R_{\beta a}=0$, whenever $\beta \epsilon S, \beta \neq a$, and the corollary follows from Theorem 2 .

It is also convenient to state Corollary 2 at this point.

Corollary 2. Let $\gamma_{1}, \ldots, \gamma_{s}$ be the members of $S$. If $R_{\beta a}=0$ whenever $\alpha, \beta \in S, \alpha \neq \beta$, then $A$ has s linearly independent characteristic column vectors $x^{1}, \ldots, x^{8}$ associated wilh 0 , where $x^{j}$ satisfies (3) with $a=\gamma_{j}$.

Iroof. Theorem 2 shows the existence of the characteristic vectors $x^{j}, j=1, \ldots, s$, satisfying (3) with $a=\gamma_{j}$. Suppose that $\sum_{h=1}^{8} \lambda_{h} x^{h}=0$. Then, for $i=1, \ldots, k$, we have $\sum_{h=1}^{8} \lambda_{h} x_{i}^{h}=0$. Let $a=\gamma_{j}$. Since $R_{\beta a}=0$ when $\beta=\gamma_{h}, h \neq j$, it follows that $x_{u}^{h}=0$ if $h \neq j$. Hence $\lambda_{j} x_{a}^{j}=0$; and $x^{j} \neq 0$ now implies $\lambda_{j}=0$. The linear independence of $x^{1}, \ldots, x^{8}$ follows. 
The Elementary Divisors, associated with 0, of a Singular 113 M-MATRIX of $x$.

5. If $x=\left(x_{1}, \ldots, x_{k}\right)$ we shall call $x_{i}$ the $i$ th vector component

Lemma 3. Let $A=\left[A_{i j}\right], i, j=1, \ldots, k$, be a singular $M$-matrix in slandard form. Let $\gamma_{1}, \ldots, \gamma_{8}$, where $\gamma_{j-1}<\gamma_{j}$, be the members of the set $S$ of indices of singular $A_{i i}$. If there exist $m$ linearly independent characteristic vectors of $A$ associated with 0 , then for each integer $n, n \leqq m$, there are at least $n$ of these vectors such that the ith vector component is non-zero for some $i \leqq \gamma_{n+s-m}$.

Proof. If $\gamma_{n+s-m}=k$ there is nothing to prove. So let $\gamma_{n+s-m}<k$ and suppose that $x^{1}, \ldots, x^{m}$ are linearly independent characteristic rectors associated with 0 , such that $x_{i}^{j}=0$, for $i=1, \ldots$, $\gamma_{n+8-m}^{\dot{a}}$ and $j=n, \ldots, m$. If $\mu=\gamma_{n+s-m}+1$, the vectors $\left(x_{\mu}^{j}, \ldots, x_{k}^{j}\right)$, $j=n, \ldots, m$, form $n-m+1$ linearly independent characteristic vectors associated with 0 of the matrix $B=\left[A_{i j}\right], i, j=\mu, \ldots, k$. But the multiplicity of 0 in $B$ equals the number of singular $A_{i i}$ in $B$, and so equals $m-n$. This yields a contradiction, and the lemma follows.

Lemma 4. Let $A=\left[A_{i j}\right], i, j=1, \ldots, k$, be a singular $M$-matrix in standard form. Lel $\gamma_{1}, \ldots, \gamma_{s}$, where $\gamma_{j-1}<\gamma_{j}$, be the members of $S$. If $A$ has s linearly independent characteristic column vectors associated swith 0 , then there exists a set $x^{1}, \ldots, x^{8}$ of such vectors for which

but

$$
\left.\begin{array}{ll}
x_{i}^{j}=0 & \text { if } i<\gamma_{j} \\
x_{i}^{j} \neq 0 & \text { if } i=\gamma_{j}
\end{array}\right\}
$$

for $j=1, \ldots, s$.

Proof. Let $z^{1}, \ldots, z^{s}$ be linearly independent characteristic vectors associated with 0 . If $z_{i}^{j}=0$ if $i<\delta_{j}$ but $z_{i}^{j} \neq 0$ if $i=\delta_{j}$, then $A_{i i} z_{i}^{j}=0$ for $i=\delta_{j}$. Hence $\delta_{j} \in S$. Thus $z_{i}^{j}=0$ if $i<\gamma_{1}$, for $j=1, \ldots, s$. It also follows from Lemma 3 , with $m=s, n=1$, that for some $j$ we have $z_{i} \neq 0$, if $i=\gamma_{1}$. We may therefore assume inductively that we have linearly independent characteristic vectors $x^{1}, \ldots, x^{n}, z^{n+1}, \ldots, z^{s}$, associated with 0 , such that (a) (8) holds for $j=1, \ldots, n$ and (b) $z_{i}^{j}=0$ if $i<\gamma_{n}$, for $j=n+1, \ldots, s$. Let $a=\gamma_{n}$. Then $A_{a a} x_{a}^{n}=A_{a \alpha} z_{a}^{j}=0, j=n+1, \ldots, s$. Since an irreducible singular 
M-matrix has only one linearly independent characteristic column vector associated with 0 , it follows that $z_{a}^{j}=\lambda_{j} x_{a}^{n}, j=n+1, \ldots, s$. Let $x^{j}=z^{j}-\lambda_{j} x^{n}, j=n+1, \ldots, s$. The vectors $x^{1}, \ldots, x^{8}$ are linearly independent characteristic vectors associated with 0 , and, for $j=n+1, \ldots, s, x_{i}^{j}=0$ if $i \leqq \gamma_{n}$. Hence by the remark at the beginning of the proof, $x_{i}^{j}=0$ if $i<\gamma_{n+1}$. for $j=n+1, \ldots, s$. It follows from Lemma 3 that there is a $j \geqq n+1$ such that $x_{i}^{j} \neq 0$, if $i=\gamma_{n+1}$. Suppose this $j=n+1$. Then (a) (8) holds for $j=1, \ldots, n+1$, and $(b) x_{i}^{j}=0$ if $i<\gamma_{n+1}$, for $j=n+2, \ldots, s$. The lemma follows by induction.

6. The following lemma is of some interest in itself. It is related to a theorem of Collatz [1], and other results on positive irreducible matrices.

Lемма 5. Let $A$ be an irreducible singular $M$-matrix and let $A x(\geqq 0$ or 0$) \geqq A x$. Then $A x=0$.

Proof. Let $A x(\geqq 0$ or $A x \leqq)$, and let $u^{\prime}$ be the strictly positive characteristic row vector of $A$, associated with 0 . If either $z<>0$ or $z<) 0$, then cither $u^{\prime} z>0$ or $u^{\prime} z<0$. Hence $A x=0$.

We now come to one of our main theorems.

Theorem 3. Let $A=\left[A_{i j}\right], i, j=1, \ldots, k$, be a singular $M \cdot$ matrix in standard form. Let $S$ be the set of indices of singular $A_{i i}$. The elementary divisors associated with the characteristic root 0 are all linear if and only if $R_{\beta a}=0$ whenever $\alpha, \beta \in S$ and $a \neq \beta$.

Proof. Let $S$ have the $s$ members $\gamma_{1}, \ldots, \gamma_{s}$ where $\gamma_{j-1}<\gamma_{j}$. The elementary divisors associated with 0 are all linear if and only if that characteristic root has $s$ linearly independent characteristic vectors associated with it.

If $R_{\beta a}=0$ whenever $a, \beta \in S$ and $a \neq \beta$ then by Corollary 2 to Theorem 2,0 has $s$ linearly independent characteristic vectors associated with it. Suppose, conversely, that 0 has the $s$ linearly independent characteristic vectors $x^{1}, \ldots, x^{8}$ associated with it. By Lemma 3, we may assume that $x^{1}, \ldots, x^{8}$ satisfy (S). Let us assume that for some $a, \beta \in S, a \neq \beta$, we have $R_{\beta a}=1$. We shall obtain a contradiction. We may choose $a, \beta$ so that $R_{\beta a}=1, \beta>a$ and $\beta-a \leqq \beta^{\prime}-\alpha^{\prime}$ for all $\alpha^{\prime}, \beta^{\prime} \in S, a^{\prime} \neq \beta^{\prime}$, for which $R_{\beta a^{\prime}}=1$. If $\alpha \leqslant \gamma \leqq \delta \leqq \beta$ and $\gamma, \delta \in S$ then $R_{\delta \gamma}=0$ unless $\gamma=a$ and $\delta=\beta$. Let 
The Elementary Divisors, Associated with 0, of a Sisgular 115 M-MATRIX

$B$ be the matrix $\left[A_{i j} \mathrm{j}, i, j=a, \ldots, \beta-1\right.$. Let $\delta_{1}, \delta_{2}, \ldots, \delta_{r}$ be the indices of the singular $A_{i i}$ of $B$ in ascending order of magnitude. Thus $\delta_{1}=a=\gamma_{j}$, say. We deduce from Corollary 2 to Theorem 2 that $B$ has $r$ linearly independent characteristic vectors $\left(z_{a}^{h}, \ldots, z_{\beta-1}^{h}\right)$, $h=1, \ldots, r$, associated with 0 , where $z_{i}^{h}$ satisfies (3) (provided we replace $a$ by $\delta_{h}$ there), for $i=a, \ldots, \beta-1$. Since the multiplicity of 0 in $B$ is $r$, any characteristic vector of $B$ associated with 0 is a linear combination of these. Since $a=\gamma_{j},\left(\underset{a}{x^{j}}, \ldots, x_{\beta-1}^{j}\right)$ is a characteristic vector of $B$ associated with 0 . Hence $x_{i}^{j}=\sum_{h=1}^{r} \lambda_{h} \vartheta_{i}^{h}$, for $i=\alpha, \ldots, \beta-1$. Further, $\lambda_{1} \neq 0$ since $x_{a}^{j} \neq 0$ but $z_{a}^{h}=0$ if $h=2$, $\ldots, r$. It follows that $A_{\beta 3} x_{\beta}^{j}=\sum_{h=1}^{r} \lambda_{h} y_{\beta}^{h}$ where $y_{\beta}^{h}=-\sum_{i=a}^{\beta-1} A_{\beta i} i_{i}^{h}$, $h=1, \ldots, r$. On putting $z_{i}^{h}=0$ when $i=1, \ldots, a-1$, we obtain from Lemma 2 that $y_{\beta}^{h}\left(>0\right.$ if $R_{\beta \gamma}=1$, but $y_{\beta}^{h}=0$ if $R_{\beta \gamma}=0$, where $\gamma=\delta_{h}$. Hence $y_{\beta}^{1}\left(>0\right.$, but $y_{\beta}^{h}=0$, for $h=2, \ldots, r$. Thus $A_{\beta \beta} x_{\beta}^{j}=\lambda_{1} y_{\beta}^{\prime}$, and so either $A_{\beta \beta} x_{\beta}^{j}\left(>0\right.$ or $0\left(>A_{\beta 3} x_{\beta}^{j}\right.$. But this is not possible, by Lemma 5. It follows that $R_{\beta a}=0$ whenever $a, \beta \in S$ and $a \neq \beta$. The theorem is proved.

In view of Theorem 1 we obtain immediately

Corollary 1. The elementary divisors associated with 0 are all linear if and only if for each $\alpha \in S$ there exists a permutation $\sigma$ of $(1, \ldots, k)$ such that $\left[A_{\sigma(i) \sigma(j)}\right], i, j=1, \ldots, k$, is in standard form, and $\sigma(\beta) \leqq \sigma(\alpha)$ for any $\beta \in S$.

The square matrix $A=\left[a_{i j}\right]$ is called Minkowskian if (1) $a_{i i} \geqq 0$ for all $i$, (2) $a_{i j} \leqq 0$, when $i \neq j$, and (3) $\sum_{j} a_{i j} \geqq 0$ for all $i$. $\mathbf{A}$ Minkowskian matrix is an M-matrix : cf. [4], [6].

Совоllary 2. Let $A$ be a singular Minkowskian matrix. The elementary divisors associated with 0 are all linear.

Proof. We may assume that $A=\left[A_{i j}\right], i, j=1, \ldots, k$, is in standard form. Let $S$ be as above. If $C=\left[c_{i j}\right]$ is an irreducible Minkowskian matrix, then $C$ is singular if and orily if $\sum_{j} c_{i j}=0$ for all $i$, [4]. Hence if $a \in S$, then $A_{n j}=0$, provided that $j \neq a$. ' It follows that $R_{a j}=0$, if $j \neq a$. The corollary now follows from Theorem 2. 
In the case when $\underset{j}{\sum} a_{i j}=0$ for all $i$, this result has already been proved by Ledermann [3].

7. Results similar to those we have found for characteristic column vectors may be stated for characteristic row vectors. Let $A=\left[A_{i j}\right], i, j=1, \ldots, k$, be in standard form. The transposed matrix $A^{\prime}=\left[A^{\prime}{ }_{i j}\right], i, j,=1, \ldots, k$ (where $\left.A^{\prime}{ }_{i j}=\left(A_{j i}\right)^{\prime}\right)$ is not necessarily in standard form. However, if $\sigma$ is the permutation for which $\sigma(i)=k+1-i, i=1, \ldots, k$, then $B=\left[A_{\sigma(i) \sigma(j)}^{\prime}\right], i, j=1, \ldots, k$, is in standard form. Let $P, P^{\prime}$, and $Q$ be the partition described above of $A, A^{\prime}$, and $B$ respectively. Then $R_{\left.\sigma(j) \sigma^{\prime} i\right)}(B, Q)=$ $R_{j i}\left(A^{\prime}, P^{\prime}\right)=R_{i j}(A, P)$. To any characteristic row vector $\left(u_{1}^{\prime}, \ldots, u_{k}^{\prime}\right)$ of $A$, associated with 0 , there corresponds the characteristic column vector $\left(u_{\sigma(1), \ldots,} u_{\sigma(k)}\right)$ of $B$ associated with 0 . We may deduce Theorem $2 a$ and Corollary $2 a$, from Theorem 2 and Corollary 2.

Theorem $2 x$. Let $A=\left[A_{i j}\right], i, j=1, \ldots, k$, be a singular $M$-matrix in standard form. Let $S$ be the sel of indices of singular $A_{i i}$. If $a \in S$ and $R_{c \beta}=0$ whenever $\beta \epsilon S$ and $\beta \neq \alpha$, then there exists a positive characteristic row vector $u^{\prime}=\left(u_{1}^{\prime}, \ldots, u_{k}{ }_{k}\right)$ associated with 0 , satisfying

for $i=1, \ldots, k$.

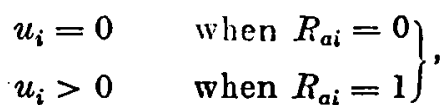

Corollary 2a. Let $\gamma_{2}, \ldots, \gamma_{s}$ be the members of $S$. If $R_{a \beta}=0$ whenever $a, \beta \in S$ and $\alpha \neq \beta$ then $A$ has s linexrly independent charac'eristic row vectors $u^{\prime 1}, \ldots, u^{\prime s}$ associated with 0 , where $u^{\prime j}$ satisfies (9) with $a=\gamma_{j}$.

Theorem 4. Let $A$ be a singular M-matrix. If the elementary divisors associated with the characteristic root 0 are all linear, then the principal idempotent element associated with 0 is positive.

Proof. Let $C$ be a matrix whose characteristic root $\omega$, of multiplicity $s$, has only linear elementary divisors associated with it. There exist linearly independent characteristic column vectors $x^{1}, \ldots, x^{8}$ and linearly independent characteristic row vectors $u^{\prime 1}, \ldots, u^{\prime s}$ associated with $\omega$, such that $a \iota^{\prime h} x^{j}=\delta_{h j}, h, j=1, \ldots, s$, the Kronecker delta. The principal idempotent element associated with $\omega$ is the matrix $\underset{h=1}{\stackrel{1}{n}} x^{h} u^{\prime h}$. 


\section{The Elementary Divisors, Assogiated with 0, of a Sisgdlar 117 M-MATRIX}

Let $P^{*}$ be the partition $A^{*}=\left[A_{i j}^{*}\right], i, j=1, \ldots, l$, of a standard form of the singular M-matrix $A$ with only linear elementary divisors associated with 0 , and let $S=\left(\gamma_{1}, \ldots, \gamma_{8}\right)$ be the set of indices of singular $A_{i i}^{*}$. By Theorem 3, $R_{\beta a}^{*}=R_{\beta u}\left(A^{*}, P^{*}\right)=0$, when $a, \beta \epsilon S, a \neq \beta$. Hence by Corollary 2 to Theorem 2 there exist linearly independent characteristic column vectors $x^{1}, \ldots, x^{s}$ associated with 0 such that $x^{j}$ satisfies (3) with $a=\gamma_{j}$. Similarly, by Corollary $2 a$ to Theorem $2 a$ there exist linearly independent characteristic row vectors $u^{\prime 1}, \ldots, u^{\prime \prime}$, associated with 0 , such that $u^{\prime j}$ satisfics (9) with $a=\gamma_{j}$. Let $a=\gamma_{j}$ and $\beta=\gamma_{h}$. Since $u^{\prime h} x^{j}=\sum_{i=1}^{k} u_{i}^{h} x_{i}^{j}$ and $u_{i}^{h} x_{i}^{j}=0$ if and only if $R_{\beta i}^{*} R_{i a}^{*}=0$ it foilows that $u^{\prime h} x^{j} \geqslant 0$, and that $u^{\prime h} x^{j}=0$ if and only if $\sum_{i=1}^{k} I_{p_{2}}^{*} R_{i a}^{*}=0$. But wo may deduce from (1) that $\sum_{i=1}^{k} R_{\beta i}^{*} R_{i a}^{*}=0$ if and only if $R_{\beta a}^{*}=0$. Hence $u^{h} x^{j}=0$ if $h \neq j$, but $u^{\prime j} x^{j}>0$, for $h, j=1, \ldots, s$. We may clearly assume that $u^{\prime 1}, \ldots, u^{\prime} s$ have been multipled by positive factors so that $u^{\prime h} x^{j}=\delta_{h j}$. Then the idempotent element of $A^{*}$ associated with 0 is $E^{*}=\sum_{j=1}^{\infty} x^{j} u^{j}$. Thus $E^{*}(\geqq 0$. If $E$ is partitioned conformably with $A$, then $E_{i i}=\sum_{h=1}^{s} x_{i}^{h} u_{i}^{\prime h}, i=1, \ldots, k$. Since $u_{a}^{\prime j}>0$, and $x_{a}^{j}>0$, but $u_{a}^{\prime h}=0$, and $x_{a}^{h}=0$, when $h \neq j$, it follows that $E_{a a}^{*}=x_{a}^{h} u_{a}^{\prime h}>0$. Hence $E^{*}(>0$.

The principal idempotent element $E$ of $A$ associated with 0 is obtained from that of $A^{*}$ by means of a transformation by a permutation matrix. Hence $E$, too, is positire.

8. Wo have already remarked that tho elementary divisors of a matrix $A$, associated with the characteristic root 0 of multiplicity $s$, are all linear if and only if there are $s$ linearly independent characteristic vectors associated with 0 . In the next two sections we shall discuss the other extreme case when there is only one elementary divisor associated with the characteristic root 0 of a singular $M$-matrix. Equivalent conditions are $(a)$ that 0 has only one linearly independent characteristic vector associated with it; or $(b)$ that thero exists a set $x^{1}, \ldots, x^{s}$ of column vectors such that

$$
A x^{j}=x^{j+1}, j=1, \ldots, s-1 ; A x^{s}=0, \text { and } x^{s} \neq 0,
$$

where $s$ is again the multiplicity of 0 . 
Lemma 6. Let $A=\left[A_{i j}\right], i, j=1, \ldots, k$, be a singular $M$-matrix in standard form. Let $S=\left(\gamma_{1}, \ldots, \gamma_{s}\right)$, where $\gamma_{j-1}<\gamma_{j}$, be the sel of indices of singular $A_{i i}$. If $x^{1}, \ldots, x^{8}$ is a set of column vectors salisfying (10), then $x^{j}, j=1, \ldots, s$, satisfies (8).

Proof. For $j=1, \ldots, s$, let $x_{i}^{j}=0$ if $i<\delta_{j}$, but $x_{i}^{j} \neq 0$ if $i=\delta_{j}$. Since $A x^{s}=0$, it follows, as in the proof of Lemma 4, that $\delta_{g} \in S$. Let us assume inductirely that $(a) \varepsilon_{j} \in S$ for $j=h, \ldots, s$; and that $(b)$ $\delta_{j-1}<\delta_{j}$ for $j=h+1, \ldots, s$. These assumptions hold for $h=s$. If $y_{i}^{j}$ satisfies (4), when $x_{i}=x_{i}^{j}$, then $A x^{j}=x^{j+1}$ if and only if

$$
A_{i i} x_{i}^{j}=y_{i}^{j}+x_{i}^{j+1} \text {, }
$$

for $i=1, \ldots, k$. I.et $\beta=\delta_{h}$. Then $y_{p}^{h}=0$; and we may deduco from (b) that $x_{\beta}^{h+1}=0$. Hence (11) holds for $i=\beta$ and $j=h$ if and only if $A_{\beta \beta_{\beta}} x_{\beta}^{h}=0$. Hence either $x_{\beta}^{h}>0$ or $x_{\beta}^{h}<0$. But $A_{\beta \beta} x_{\beta}^{h-1}=y_{\beta}^{h-1}+x_{\beta}^{h}$. It follows by Lcmma 5 that $(a)$ and $(b)$ imply that $y_{\beta}^{h-1} \neq 0$. Thus $\delta_{h-1}<\beta=\delta_{h}$. We deduce that if $a=\delta_{h-1}$ then

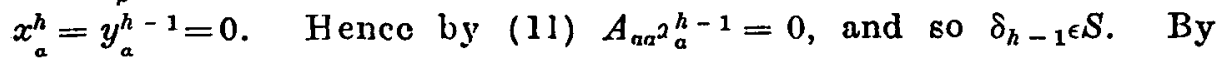
induction we obtain that $\delta_{j}=S, j=1, \ldots, s$, and that $\delta_{j-1}<\delta_{j}, j=2, \ldots, s$. Hence $\delta_{j}=\gamma_{j}, j=1, \ldots, s$ and the lemma is proved.

9. Lenma 7. Let $A$ be an irreducible $M$-matrix. Let $z$ and $y$, where $y$ (> 0 , be column vectors conformable with $A$. Then there exist a real $\lambda$ and a column vector $x$ such that $A x=\lambda y+z$.

Proof. If $A$ is non-singular, then there cxists such an $x$ for any $\lambda$. Suppose $A$ singular. Since 0 is a simple characteristic root of $A$, the nullity of $A$ is 1 . By Lemma 5, $y$ is linearly independent of the columns of $A$. Hence any column vector is a linear combination of the columns of $A$ and $y$. The lemma follows.

Theorem 5. Let $A=\left[A_{i j}\right], i, j=1, \ldots, k$, be a singular $M$-matrix in standard form. Lct $S$ be the set of indices of singular $A_{i i}$. There is only one elementary divicor associaled with the characteristic root 0 of $A$ if and only if $R_{\beta a}=1$, whenever $a, \beta \in S$ and $\beta>a$.

Proof. Suppose that there is only one elementary divisor associated with 0 . Let $x^{1}, \ldots, x^{s}$ be a set of column vectors satisfying (10). By Lemma 6, $x^{j}$ satisfies (8), $j=1, \ldots, s$. Let $a=\gamma_{h-1}, \beta=\gamma_{h}$. The 
conditions $(a)$ and $(b)$ of the proof of Lemma 6 are clearly satisfied, and so $y_{\beta}^{h-1}=-\sum_{i=1}^{\beta-1} A_{i i} x_{i}^{h-1}=-\sum_{i=a}^{\beta} A_{i i} x_{i}^{h-1} \neq 0 . \quad \operatorname{But}\left(x_{a}^{h-1}, \ldots, x_{\beta-1}^{h-1}\right)$ is the one linearly independent characteristic column vector associated with 0 of $B=\left[A_{i j}\right], i, j=a, \ldots, \beta-1$, since $x_{i}^{h}=0$, for $i=a, \ldots, \beta-1$. It therefore follows from Theorem 2 that $x_{i}^{h}, i=a, \ldots, \beta-1$, satisfies (3), provided that $x^{1}, \ldots, x^{s}$ have been multiplicd by -1 if necessary. Therefore $x_{i}^{h}, i=1, \ldots, \beta-1$, satisfy (3). Hence, by Lemma 2, it follows from $y_{\beta}^{h-1} \neq 0$ that $R_{\beta a}=1$, when $a=\gamma_{h-1}, \beta=\gamma_{h}$. This is a particular case of the required result. To deduce the general case, let $a=\gamma_{j}, \beta=\gamma_{h}$, where $h>j$. Then $R_{\beta a} \geqslant R_{\gamma_{h} \gamma_{h-1}} \ldots R_{\gamma_{j+1} \gamma_{j}}=1$. Hence $R_{\beta a}=1$, whenever $a, \beta \in S^{\prime}$ and $\beta>\alpha$.

Conversely, let us suppose that $R_{\beta a}=1$ whenever $a, \beta \in S$ and $\beta>a$. By Theorem 2, Corollary 1, there exists a characteristic column vector $x^{8}$, associated with 0 , which gatisfies (8). Let us suppose that there exist column vectors $x^{j}$ satisfying $(8), j=h, \ldots, s$, such that

$$
A x^{j}=x^{j+1}, j=h, \ldots, s-1, A x^{s}=0, x^{8} \neq 0 .
$$

We shall construct a vector $x^{h-1}$ satisfying (8) and $A x^{h-1}=x^{h}$. Let $a=\gamma_{h-1}, \beta=\gamma_{h}$. Let $x_{i}^{h-1}=0, i=1, \ldots, a-1$, and let $\left(x_{\alpha}^{h-1}, \ldots, x_{\beta-1}^{h-1}\right)$ be the one linearly independent characteristic vector, associated with 0 , of $B=\left[A_{i j}\right], i, j=a, \ldots, \beta-1$. Then $x_{i}^{h-1}, i=1$, $\ldots, \beta-1$, satisfies (3) provided that the $x_{i}^{h-1}$ have all been multiplied by -1 , if necessary. If, for $i=1, \ldots, \beta, y_{i}^{h-1}$ is chosen to satisfy (4) with $x$ replaced there by $x^{h-1}$, then it follows by Lemma 2 that $y_{\beta}^{h-1}\left(>0\right.$. Hence by Lemma 7, there exist a $\lambda$ and an $x_{\beta}^{h-1}$ such that $A_{\beta \beta} x_{\beta}^{h-1}=\lambda y_{\beta}^{h-1}+x_{\beta}^{h}$. We now write $x_{i}^{h-1}$ for $\lambda x_{i}^{h-1}, i=1, \ldots$, $\beta-1, \lambda y_{i}^{h-1}$ for $y_{i}^{h-1}, i=1, \ldots, \beta$, and leave $x_{\beta}^{h-1}$ unchanged. Since $x_{i}=0$, when $i<\beta, x_{i}^{h-1}, i=1, \ldots, \beta$ satisfies (11) with $j=\grave{n}-1$.

Let us assume inductively that $x_{i}^{h-1}, i=1, \ldots, l-1$, satisfies (11), where $l>\beta$. We must consider two cases: $l \notin S$ and $l \in S$. If $l \notin S$, then (11) is satisfied when $x_{l}^{h-1}=A_{l l}^{-1}\left(y_{l}^{h-1}+x_{l}^{h}\right)$. If $l_{\epsilon} S$, say $l=\gamma_{h+m}$ (where clearly $m>0$ ) then there exist, by Lemma 7, a $\lambda$ and an $x_{l}^{h-1}$ for which $A_{l l} x_{l}^{h-1}=y_{l}^{h-1}+x_{l}^{h}+\lambda x_{l}^{h+m}$, since either $x_{l}^{h+m}>0$ or $x_{l}^{h+m}<0$. We now replace $x^{j}$ by $x^{j}+\lambda x^{j+m}, j=h, \ldots, s$, where, by conrention, $x^{j+m}=0$ if $j+m>s$. Then $x^{h}, \ldots, x^{s}$ again satisfy (12) and since the original $x_{i}^{h+m}=0$ when $i<l$, it follows that $x_{1}^{h-1}, \ldots, x_{l}^{h-1}$ 
satisfy (11) with $j=h-1$. By induction we obtain a vector $x^{h-1}=\left(x_{1}^{h-1}, \ldots, x_{k}^{h-1}\right)$ satisfying (11) with $j=h-1$. Thus in addition to (12) we have $A x^{h-1}=x^{h}$, where $x^{h-1}$ satisfies (8). Using induction again we obtain a set of vectors satisfying (10) and the theorem is proved.

In virtue of Theorem 1 we obtain the following corollary.

Conollary. Let $\gamma$ be the largest member of $S$. There is only one elementary divisor associated with the characleris!ic root 0 of $A$ if and only if for any permutation $\sigma$ of $(1, \ldots, k)$ for which $\left[A_{\sigma(i) \sigma(j)}\right], i, j=1, \ldots, k$, is in slandard form, we have $\sigma(\gamma) \geqslant \sigma(\alpha)$ whenever $a \in S$.

An argument along the lines of the second half of the proof of Theorem 5 would lead to the following result, which we shall enunciate as a theorem, though we shall omit the proof. First we should have to generalise Lemma 2.

Theorem 6. Let $A=\left[A_{i j}\right], i, j=1, \ldots, k$, be a singular $M$-matrix in standard form. Let $S=\left(\gamma_{1}, \ldots, \gamma_{s}\right), \gamma_{j-1}<\gamma_{j}$, be the set of indices of singular $A_{i i}$. If there is only one elementary divisor associated with 0 then there exist positive column vectors $z^{1}, \ldots, z^{s}$ such that $x^{1}, \ldots, x^{8}$ satisfy (10) if $x^{j}=(-1)^{j} z^{j}, j=1, \ldots, s$. In fact

$$
z_{i}^{h}=0 \text { when } \sum_{j=h}^{s} R_{i \gamma_{j}}=0,
$$

and

$$
z_{i}^{h}>0 \text { when } \sum_{j=h}^{s} R_{i \gamma}>0 .
$$

The standard forms of $A$ depend only on the set $\Sigma$ of index pairs of non-zero coefficients of $A$. It is clearly decided by $\Sigma$ and $S$ whether (a) $R_{\beta a}=0$, whenever $\alpha, \beta \in S$, and $\alpha \neq \beta$; or $(b) R_{\beta a}=1$, whenever $a, \beta \epsilon S$ and $\beta>\alpha$. Let $P$ be a positive matrix, with largest characteristic root $\rho$. The degrees of the elementary divisors of $P$ associated with $\rho$ are the same as those of the clementary divisors, associated with 0 , of the M-matrix $\rho I-P$. Hence Theorems 3 and 5 fuifil the claims of $\S 1$.

10. Let $A=\left[A_{i j}\right], i, j=1, \ldots, k$, be a singular M-matrix in standard form. Let $S=\left(\gamma_{1}, \ldots, \gamma_{s}\right), \gamma_{j-1}<\gamma_{j}$, be as usual, the set of indices of singular $A_{i i}$. If $s=1$, then there is clearly only one elementary divisor, associated with 0 , and it is linear. If $s=2$, then there are two elementary divisors of degree 1, or one of degrce 2, associated with 0 , according as $R_{\beta a}=0$ or $R_{\beta a}=1$, where $\alpha=\gamma_{1}$, and $\beta=\gamma_{2}$. Suppose that $s=3$ and let $\gamma_{1}=a, \gamma_{2}=\beta$ and $\gamma_{3}=\gamma$. Since 
The Elementary Divisors, Associated with 0, of a Singular 121 M-MaTRIX

$R_{a \beta}=R_{a y}=R_{\beta \gamma}=0$, and since $R_{\gamma \beta}=R_{\beta \alpha}=1$ implies that $R_{\gamma a}=1$, we may consider seven cases according as $R_{\gamma \beta}, R_{\gamma^{a}}, R_{\beta a}$ are 1 or 0 . The cases $R_{\gamma \beta}=R_{\gamma a}=R_{\beta a}=0$, and $R_{\gamma \beta}=R_{\gamma \alpha}=R_{\beta 2}=1$, are covered by Theorems 3 and 5 . We dedluce that in the five other cases 0 must have associated with it one elementary divisor of degree 1 and one of degree 2. Tuis means that $A$ has two linearly independent column vectors $z^{1}, z^{2}$ associated with 0 , and that there exists a vector $x$ satisfying either $A x=z^{1}$ or $A x=z^{2}$. By considering each of the five cases separately it is possible to demonstrate the existence of these vectors without any appeal to Theorems 3 and 5 .

As an example we shall consider the case $R_{y \beta}=R_{\gamma a}=1, R_{\beta a}=0$. Though we shall use the theorems proved previously it would be possible to use special cases of these results which could be proved more simply. It follows from Theorem 5 , applicd to $\left[A_{i j}\right], i, j=\beta, \ldots, k$, that $A$ has a characteristic vector $x^{3}$, associated with 0 , for which $x_{i}^{3}=0$ when $i<\gamma$, and that there exists an $x^{2}$ satisfying $A x^{2}=x^{3}$. Let $\left(:_{1}^{j}, \ldots, z_{\gamma-1}^{j}\right), j=1,2$, be the two linearly independent characteristic column vectors, associated with 0 , of $\left[A_{i j}\right], i, j=1, \ldots, \gamma-1$. The existence of these vectors is shown by 'Theorem 3. By Theorem 1, Corollary 2, we may assume that $z_{i}^{j}=0$ if $R_{i \gamma_{j}}=0$ and $z_{i}^{j}>0$ if $R_{i \gamma_{j}}=1$, for $j=1,2$. Hence, by Lemma $2, w_{\gamma}^{j}\left(>0, j=1,2\right.$, where $w_{\gamma}^{j}=-\sum_{h=1}^{\gamma-1} A_{h h j}$. By Lemma $\bar{i}$, there exist $a \lambda$ and an $x_{\gamma}^{1}$ satisfying

$$
A_{\gamma \gamma} x_{\gamma}^{1}=w_{\gamma}^{1}+\lambda w_{\gamma}^{2} \text {. }
$$

Let $x_{i}^{1}=z_{i}^{1}+\lambda z_{i}^{2}, i=1, \ldots, \gamma-1$. Then $\left(x_{1}^{1}, \ldots, x_{\gamma}^{1}\right)$ is nonzero and, for $i=1, \ldots, \gamma, x_{i}^{1}$ satisfies (7) provided $y_{i}^{1}$ is chosen to satisfy (4). Since $A_{i i}$ is non-singular if $i>\gamma$, it is easy to establish the existence of a vector $x^{1}=\left(x_{1}^{1}, \ldots, x_{k}^{1}\right)$ where $x_{i}^{1}$ satisfies (i) for $i=1, \ldots, k$. Thus $x^{1}$ and $x^{3}$ are characteristic column vectors associated with 0 , which are linearly independent as $x_{a}^{1}=z_{a}^{1}+\lambda z_{a}^{2}=z_{a}^{1}>0$, while $x_{a}^{3}=0$; and $A x^{2}=x^{3}$.

It follows from Lemma 5 that $\lambda<0$ in (13). Hence $x_{\beta}^{1}=z_{\beta}^{1}+\lambda z_{\beta}^{2}=$ $\lambda z_{\beta}^{2}<0$, but $x_{\alpha}^{1}>0$, as already noted. Hence $x^{1}$ is neither positive nor negative. It is easily established that this property is shared by one of any two linearly independent characteristic vectors, associated 
with 0 , of the matrix $A$ we have been considering. It is due to this that $S$ and $\Sigma$ do not necessarily completely determine the elementary divisors associated with 0 of a singular M-matrix when $s>3$. Thus, in both $B$ and $C$ below, $k=4, S=(1,2,3,4), s=4$ and $R_{43}=R_{21}=0$, $R_{42}=R_{41}=R_{32}=R_{31}=1$.

$$
B=\left[\begin{array}{cccc}
\cdot & \cdot & \cdot & \cdot \\
\cdot & \cdot & \cdot & \cdot \\
-1 & -1 & \cdot & \cdot \\
-1 & -1 & \cdot & \cdot
\end{array}\right] \quad C=\left[\begin{array}{rrrr}
\cdot & \cdot & \cdot & \cdot \\
\cdot & \cdot & \cdot & \cdot \\
-1-1 & \cdot & \cdot \\
. & -1 & \cdot & \cdot
\end{array}\right] \text {. }
$$

But $B$ is of rank 1, $C$ of rank 2. Hence the elementary divisors associated with 0 differ for the two matrices. We also note that the principal idempotent elcment, associated with 0 , of both $B$ and $C$ is the unit matrix, which is, of course, positive. It follows that the converse of Theorem 4 does not hold.

Most of the results of this paper are contained in my $1952 \mathrm{Ph} . \mathrm{D}$. thesis, which was written under the supervision of Professor A. C. Aitken. My thanks are due to Professor Aitken for his great encouragement.

\section{REFERENCES.}

1. L. Collatz, " Kinschlieszungsatz für die charakteristischen Zahlen von Matrizen," Math. Zeitschrift, 48 (1942), 221-2:6.

2. G. Frobenius, "Ueber Matrizen aus nicht negativen Elementen," Sitzungsberichte der P,eussischen Akadtmie der Wiss:nschaften (1912), 456-477.

3. W. Ledermann, "Asymptotic probability distribution in Markoff processes," Proc. Cambridge Phil. Soc. 46 (1950), 581.594.

4. A. Ostrowski, "Ueber die Determinanten mit ueberwiegender Hauptdiagonale," Commentarii Helvelici Math., 10 (1937), 69.96.

5. - " Ueber das Nichtversch rinden einer Klasse von Determinanten und die Lokalisiervng der charakteristischen Wurzeln von Matrizen, "Compositio Math., 9 (1951), 209-226.

6. H. Schneider "An inequality for latent roots applied to determinants with dominant principal diagonal," Journal London Math. Soc., 23 (195̄), 8-20.

7. O. Taussky, "Bounds for the characteristic roots of matrices, II," Journal of Research, Nat. Bureau of Standards, 46 (1951), 124-125.

8. H. Wielandt, "Unzerlegbare, nicht-negative Matrizen," Math. Zeilschrift, $52(1950), 642 \cdot 648$.

\section{The Queen's Cniversity,}

\section{BELTAST.}

